\title{
The Effects of Reflexology on Fatigue Severity of Patients with Cancer
}

\author{
Hassan Nourmohammadi', Minoo Motaghi², Milad Borji ${ }^{3}$, Asma Tarjoman ${ }^{4}$, \\ Behrouz Soltany ${ }^{3 *}$
}

\begin{abstract}
Introduction: Breast cancer is a major threat to women's health and a common factor that can reduce their life expectancy. Complementary medicine such as reflexology is known to reduce fatigue severity in cancer patients. The present study aimed to cultivate the effect of reflexology on fatigue severity of patients with breast cancer. Methods: The present pre-post clinical trial recruited 57 patients with breast cancer and involved an experimental and a control group. All patients were livening in Ilam, Iran. Patients were randomly assigned to two groups of experimental $(\mathrm{N}=27)$ and control ( $\mathrm{N}=30)$. The experimental group received reflexology for 4 sessions. Data were collected using Fatigue severity scale (FSS) and demographic information questionnaire. FSS was completed by the patients twice; before the intervention and 2 months after the intervention. Data were analyzed using SPSS and running t-test and ANOVA. Results: Results showed no significant difference in fatigue severity between experimental $(45.44 \pm 5.30)$ and control $(43.66 \pm 7.68)$ groups prior to the intervention ( $\mathrm{p}>0.05)$. However, after conducting the intervention, a significant difference in fatigue severity was seen between the experimental (20.66 \pm 4.54$)$ and control (40.36 \pm 9.58$)$ groups $(\mathrm{p}=0.000)$. Conclusion: The present study showed that reflexology decrease fatigue severity in patients with breast cancer and community health nursing can to use of these complementary medicine to increase patients health conditions.
\end{abstract}

Keywords: Fatigue severity- reflexology- breast cancer

Asian Pac J Cancer Prev, 20 (2), 391-394

\section{Introduction}

Cancer is an important healthcare issue worldwide because it is the third leading cause of death and the second most common chronic disease (Rossi et al., 2017; Shahrbabaki et al., 2012; Khoshnood et al., 2018; Safizadeh et al., 2018). In fact, estimated deaths and complications caused by cancer are on rise (Organization, 2015; Khoshnood et al., 2018; Pourdeghatkar et al., 2017). Breast cancer is a major threat in particular to women's health and a common risk factor that can decrease life expectancy among sufferers (Parkin et al., 1999). In Iran, many women deaths was reported due to breast cancer (Reyhani et al., 2012). One common symptom among patients with breast cancer is fatigue (Reinertsen et al., 2010), 70\% of which is caused by chemotherapy and radiotherapy (Rad et al., 2016).

Previous studies have indicated that fatigue decreases the quality of life in patients with cancer that is associated with lower survival rate and higher mortality rate. In addition, fatigue may affect patient's physical activity, increase his/her sense of loneliness and isolation, and interfere with daily living activities (Barsevick et al., 2008; Rad et al., 2016). In recent years, non-drug therapies have been used in combination with drug therapy to decrease fatigue in patients with cancer (Mustian et al., 2011). Patients themselves can try complementary medicine, which increases their independence. Furthermore, in comparison with drug therapy, complementary medicine has lower rates of negative side effects and consequences (Zargarzadeh and Memarian, 2013). Consequently, complementary medicine is becoming more popular globally. In most regions of Africa, Asia, and Latin America, it is among the most important methods of care and treatment available to patients (Zargarzadeh and Memarian, 2013).

Common interventions suggested to reduce fatigue are yoga (Vadiraja et al., 2017; Sadja and Mills, 2013), cognitive-behavioral therapy (Sandler et al., 2017, Montgomery et al., 2014), acupressure (Bastani et al., 2015), exercise (Shariati et al., 2010), and reflexology (Jin and Kim, 2005; Özdelikara and Tan, 2017). Reflexology is a treatment in which practitioners apply pressure to the feet or hands using their fingers. It is used to treat various

${ }^{1}$ Medical Oncologist and Hematologist, Department of Internal Medicine, Ilam University of Medical Science, Ilam, ${ }^{2}$ Nursing Department, Faculty of nursing and Midwifery, Isfahan (Khorasgan) Branch, Islamic Azad University, Isfahan, ${ }^{3}$ Department of Nursing, Faculty of Nursing and Midwifery, ${ }^{4}$ Students Research Committee, Kermanshah University of Medical Science, Kermanshah, Iran.*For Correspondence: soltanybehrouz@yahoo.com 
symptoms, such as pain, anxiety, and fear (Valiani et al., 2010; Özdemir et al., 2013; Hudson et al., 2015; Hughes et al., 2009; Bagheri-Nesami et al., 2014). This therapy increases patient comfort by inducing physiological changes. Specifically, the pressure on the reflexive areas stimulates hundreds of nerve endings in the soles, causing the release of endorphins, and consequently prevents pain transfer, causes comfort and anesthesia, decreases tension, and increases tranquility (Pitman and MacKenzie, 2002). Given high prevalence of breast cancer in Iran and the importance of complementary medicine in enhancing patients' health, this study aimed to investigate the effect of reflexology on fatigue severity in patients with breast cancer.

\section{Materials and Methods}

The present investigation was a pre-post, double-blind study, involving experimental and control groups. The study was conducted on patients with breast cancer living in Ilam, Iran, during at least previous 8 month. A total of 60 patients with a definitive breast cancer diagnosis were randomly recruited for the study. All the patients were in the first stage of breast cancer and received chemotherapy. The inclusion criteria were as follows: (1) definitive breast cancer diagnosis, (2) absence of metastasis according to oncologist' diagnosis, (3) absence of cardiovascular disease, (4) absence of bone fractures, skin lesions, or disorders, (5) absence of mental disorders, (6) absence of diabetes mellitus, (7) having cancer for at least one year, having contact information, and (9) residing in Ilam, Iran .

The exclusion criteria were as follows: (1) non-cooperation of patients during the intervention, (2) absence for more than three sessions, (3) using other methods of complementary or alternative medicine, and (4) suffering from any crises affecting the patients' fatigue severity. The study was initiated after receiving permission from the Research Ethics Committee. The selected Patients were divided randomly into two groups of experimental group (reflexology group) and control group. To prevent information transfer between two groups, the randomization was done based on the days of week. Specifically, at the beginning of every week, we randomly selected four days and allocated them to reflexology group. During the interventions, three patients from experimental group were excluded from the study and the study was continued with 27 patients in the experimental group and 30 patients in the control group.

For data collection, demographic information questionnaire and fatigue severity scale (FSS) were used. Demographic information questionnaire has 9 questions. Totally, the scores range between 0 and 63, and higher scores reveal higher severity of fatigued (Shahid et al., 2011). Experimental group received reflexology twice a week for 4 consecutive weeks while each session lasted for 20 minutes. To prepare patients' legs before performing reflexology, the patients carried out movements such as bending the legs up and down, stretching the calves and ankles, turning the ankles inward and outward, and flexing the feet. General reflexology was performed using a worm-like movement of the thumb and index finger on reflexive areas. Specialized reflexology was then performed by pressing the major reflexive points of the soles (solar plexus, pituitary gland, spinal cord, vertebral column, pelvis, and limbs) with the thumb and index finger. The toes and other reflexive areas of the soles, as well as the back of the feet were also massaged. Pressing the thumb on the solar plexus signaled the end of the intervention (Soheili shahreza et al., 2014). Control group received routine care and the prescribed medication by the oncologist. The FSS was re-filled 2 months after the intervention.

Written informed consent was obtained from each patient and no costs were imposed upon patients for receiving reflexology. The study followed the Declaration of Helsinki and Belmont Report and research publication ethics were observed. Data were analyzed using SPSS (version 16) and running t-test and ANOVA .

\section{Results}

There was no significant difference among the 2 study groups in terms of demographic information before conducting the intervention $(\mathrm{p}>0.05)$ (Table1).

As shown in Table 2, no significant difference in fatigue severity was observed between the experimental $(45.44 \pm 5.30)$ and control $(43.66 \pm 7.68)$ groups prior to conduct the intervention $(p>0.05)$. However, after the intervention, a significant difference in fatigue severity was seen between the experimental $(20.66 \pm 4.54)$ and control $(40.36 \pm 9.58)$ groups $(\mathrm{p}=0.000)$.

Table 1. Demographic Information of Patients with Breast Cancer in the Two Study Groups

\begin{tabular}{|c|c|c|c|}
\hline \multirow[t]{2}{*}{ Variable } & \multicolumn{2}{|c|}{ Group } & \multirow[t]{2}{*}{ p-value } \\
\hline & Reflexology & Contorol & \\
\hline Age (Mean (SD)) & $47.85(8.39)$ & $50.86(6.50)$ & $\mathrm{P}=0.13$ \\
\hline Maritl status & & & $\mathrm{P}=0.89$ \\
\hline Has spouse & $24(88.9)$ & $27(90)$ & \\
\hline No spouse & $3(11.1)$ & $3(10)$ & \\
\hline Education Level & & & $\mathrm{P}=0.32$ \\
\hline Illiterate & $6(22.2)$ & $9(30)$ & \\
\hline Diploma & $16(59.3)$ & $17(56.7)$ & \\
\hline Collegiate & $5(18.5)$ & $4(13.3)$ & \\
\hline Annual Icome & & & $\mathrm{P}=0.45$ \\
\hline Weak & $22(81.5)$ & $22(73.3)$ & \\
\hline medium & $4(14.8)$ & $6(20)$ & \\
\hline Good & $1(3.7)$ & $2(6.7)$ & \\
\hline Family support & & & $\mathrm{P}=0.56$ \\
\hline Weak & $2(6.7)$ & $4(14.8)$ & \\
\hline medium & $19(63.3)$ & $10(37)$ & \\
\hline Good & $9(30)$ & $13(48.1)$ & \\
\hline \multicolumn{3}{|c|}{ Believes in the impact Palliative interventions } & $\mathrm{P}=0.13$ \\
\hline Yes & $18(66.7)$ & $14(46.7)$ & \\
\hline No & $9(33.3)$ & $16(53.3)$ & \\
\hline
\end{tabular}


Table 2. Comparison of Patients' Fatigue Severity before and after the Intervention

\begin{tabular}{lccc}
\hline Variable & $\begin{array}{c}\text { Reflexology } \\
\text { Mean (SD) }\end{array}$ & $\begin{array}{c}\text { Control } \\
\text { Mean (SD) }\end{array}$ & $\mathrm{P}, \mathrm{T}$ \\
\hline $\begin{array}{c}\text { Fatigue severity } \\
\text { Before }\end{array}$ & $45.44 \pm 5.30$ & $43.66 \pm 7.68$ & $\mathrm{P}=0.31$ \\
& & & $\mathrm{~T}=1.05$ \\
After & $20.66 \pm 4.54$ & $40.36 \pm 9.58$ & $\mathrm{P}=0.000$ \\
& & & $\mathrm{~T}=-9.73$ \\
p-value & $\mathrm{P}=0.000$ & $\mathrm{P}=0.16$ & \\
\hline
\end{tabular}

\section{Discussion}

The present study showed that reflexology decreased fatigue severity in patients with cancer, which is similar to findings reported by the previous studies, suggesting that reflexology can improve the general health of patients. For instance, Özdemir G et al., (2013) addressed fear, pain, and fatigue in patients undergoing hemodialysis and concluded that reflexology had a positive effect on their fatigue and could decrease its severity. Hughes et al., (2009) investigated pain in patients with multiple sclerosis and found that reflexology improved patients' conditions and reduced their pain and fatigue. Furthermore, various studies indicated that reflexology improved the health of cancer patients. For example, Stephenson et al., (2007) revealed that reflexology decreased pain in patients with cancer, and Tsay et al., (2008) demonstrated that the therapy ameliorated both anxiety and pain in patients with cancer.

In particular, Özdemir et al., (2013) conducted a study to determine the effect of reflexive sole massage on fatigue severity, pain, and muscle cramps in patients undergoing hemodialysis. The results suggested that reflexology decreased fatigue severity, muscle cramps, and pain in these patients. Yang et al., (2005) and Roshanravan et al., (2016) found that reflexology on the soles decreased fatigue, supporting the findings of the present study. Moreover, reflexology appears to cause physiological changes that increase comfort in patients (Jin and Kim, 2005).

In conclusion, the present study showed that reflexology decreased fatigue severity in patients with breast cancer. Therefore, community health nursing staff are suggested to use this complementary medicine to enhance patients' health conditions.

\section{Acknowledgments}

Student Research Committee, Kermanshah University of Medical Sciences, Kermanshah, Iran. (Project Code: 3007139).

\section{References}

Bagheri-Nesami M, Shorofi SA, Zargar N, et al (2014). The effects of foot reflexology massage on anxiety in patients following coronary artery bypass graft surgery: a randomized controlled trial. Complement Ther Clin Pract, 20, $42-7$.

Barsevick AM, Newhall T, Brown S (2008). Management of cancer-related fatigue. Clin J Oncol Nurs, 12, 21.

Bastani F, Khosravi M, Borimnejad L, Arbabi N (2015). The effect of acupressure on cancer-related fatigue among school-aged children with acute lymphoblastic leukemia. Iran J Nurs Midwifery Res, 20, 545.

Hudson BF, Davidson J, Whiteley MS (2015). The impact of hand reflexology on pain, anxiety and satisfaction during minimally invasive surgery under local anaesthetic: A randomised controlled trial. Int J Nurs Stud, 52, 1789-97.

Hughes C, Smyth S, Lowe-Strong A (2009). Reflexology for the treatment of pain in people with multiple sclerosis: a double-blind randomised sham-controlled clinical trial. Mult Scler, 15, 1329-38.

Jin SJ, Kim YK (2005). The effects of foot reflexology massage on sleep and fatigue of elderly women. J Korean Acad Adult Nurs, 17, 493-502.

Khoshnood Z, Iranmanesh S, Rayyani M (2018). Getting out or remaining in the cage of inauthentic self: The meaning of existential challenges in patients' with cancer. Indian $J$ Palliat Care, 24, 131-8.

Khoshnood Z, Iranmanesh S, Rayyani M, Dehghan M (2018). Body-mind healing strategies in patients with cancer: A qualitative content analysis. Asian Pac J Cancer Prev, 19, 1691.

Montgomery GH, David D, Kangas M, et al (2014). Randomized controlled trial of a cognitive-behavioral therapy plus hypnosis intervention to control fatigue in patients undergoing radiotherapy for breast cancer. J Clin Oncol, 32, 557-63.

Mustian KM, Roscoe JA, Palesh OG, et al (2011). Polarity therapy for cancer-related fatigue in patients with breast cancer receiving radiation therapy: a randomized controlled pilot study. Integr Cancer Ther, 10, 27-37.

Organization WH (2015). World Health Organization Cancer Fact Sheet.

Özdelikara A, Tan M (2017). The effect of reflexology on chemotherapy-induced nausea, vomiting, and fatigue in breast cancer patients. Asia-Pac J Oncol Nurs, 4, 241.

Özdemir G, Ovayolu N, Ovayolu Ö (2013). The effect of reflexology applied on haemodialysis patients with fatigue, pain and cramps. Int J Nurs Pract, 19, 265-73.

Parkin DM, Pisani P, Ferlay J (1999). Estimates of the worldwide incidence of 25 major cancers in 1990. Int J Cancer, 80, 827-41.

Pitman V, Mackenzie K (2002). Reflexology: a practical approach, Nelson Thornes.

pourdeghatkar F, Motaghi M, Darbandi B, Baghersalimi A (2017). Comparative effect of chamomile mouthwash and topical mouth rinse in prevention of chemotherapy-induced oral mucositis in Iranian pediatric patients with acute lymphoblastic leukemia. IJBC, 9, 84-88.

Rad M, Borzoee F, Mohebbi M (2016). The effect of humor therapy on fatigue severity and quality of life in breast cancer patients undergoing external radiation therapy. ZUMS $J, 24,102-14$.

Reinertsen KV, Cvancarova M, Loge JH, et al (2010). Predictors and course of chronic fatigue in long-term breast cancer survivors. J Cancer Surviv, 4, 405-14.

Reyhani M, Fahami F, Mosharaf S, Tarkesh N (2012). The relationship between the amount of dairy products and animal proteins consumption and breast cancer in isfahani women. ZUMS J, 20, 45-54.

Roshanravan M, Jouybari L, Taghanaki H, et al (2016). Effect of foot reflexology on fatigue in patients undergoing hemodialysis: A sham-controlled randomized trial. J Mazandaran Univ Med Sci, 26, 32-41.

Rossi E, Di Stefano M, Firenzuoli F, Monechi MV, Baccetti S 
(2017). Add-on complementary medicine in cancer care: Evidence in literature and experiences of integration. Medicines, 4, 5.

Sadja J, Mills PJ (2013). Effects of yoga interventions on fatigue in cancer patients and survivors: a systematic review of randomized controlled trials. EXPLORE: The Journal of Science and Healing, 9, 232-243.

Safizadeh H, Hafezpour S, Shahrbabaki PM (2018). Health damaged context: Barriers to breast cancer screening from viewpoint of Iranian health volunteers. Asian Pac J Cancer Prev, 19, 1941.

Sandler CX, Goldstein D, Horsfield S, et al (2017). Randomized evaluation of cognitive-behavioral therapy and graded exercise therapy for post-cancer fatigue. J Pain Symptom Manage, 54, 74-84.

Shahid A, Wilkinson K, Marcu S, Shapiro CM (2011). Fatigue severity scale (FSS). STOP, THAT and one hundred other sleep scales. Springer, pp 1-10.

Shahrbabaki PM, Farokhzadian J, Hasanabadi Z, Hojjatoleslami S (2012). The evaluation of the educational plan of breast self-examination of women referring to health centers. Procedia Soc Behav Sci, 31, 913-17.

Shariati A, Haghighi S, Fayyazi S, Tabesh H, Kalboland MM (2010). The effect of exercise on the severity of the fatigue in colorectal cancer patients who received chemotherapy in Ahwaz. Iran J Nurs Midwifery Res, 15, 145.

Soheili Shahreza M, Nazari F, Shayegannejad V, Valiani M (2014). The effects of reflexology on physical symptoms in women with multiple sclerosis. CMJA, 4, 820-30.

Stephenson NL, Swanson M, Dalton J, Keefe FJ, Engelke M (2007). Partner-delivered reflexology: effects on cancer pain and anxiety. Oncol Nurs Forum, 34, 127-32.

Tsay S-L, Chen HL, Chen S-C, Lin H-R, Lin K-C (2008). Effects of reflexotherapy on acute postoperative pain and anxiety among patients with digestive cancer. Cancer Nurs, 31, 109-15.

Vadiraja H, Rao RM, Nagarathna R, et al (2017). Effects of yoga in managing fatigue in breast cancer patients: A randomized controlled trial. Indian J Palliat Care, 23, 247.

Valiani M, Abediyan S, Ahmadi SM, Pahlavanzadeh S, Hassanzadeh A (2010). The effect of relaxation techniques to ease the stress in infertile women. Iran J Nurs Midwifery Res, 15, 259.

Yang JH (2005). The effects of foot reflexology on nausea, vomiting and fatigue of breast cancer patients undergoing chemotherapy. J Korean Acad Nurs, 35, 177-85.

Zargarzadeh M, Memarian R (2013). Assesing barriers for using of complementary medicine in relieving pain in patients by nurses. QJNM, 1, 45-53.

\section{(ब) $\odot \otimes$}

This work is licensed under a Creative Commons AttributionNon Commercial 4.0 International License. 